\title{
Efficient Functionalization of Additives at Supramolecular Material Surfaces
}

Citation for published version (APA):

Goor, O. J. G. M., Keizer, H. M., Bruinen, A. L., Schmitz, M. G. J., Versteegen, R. M., Janssen, H. M., Heeren, R. M. A., \& Dankers, P. Y. W. (2017). Efficient Functionalization of Additives at Supramolecular Material Surfaces. Advanced Materials, 29(5), [1604652]. https://doi.org/10.1002/adma.201604652

Document status and date:

Published: 02/02/2017

DOI:

10.1002/adma.201604652

Document Version:

Publisher's PDF, also known as Version of record

Document license:

Taverne

Please check the document version of this publication:

- A submitted manuscript is the version of the article upon submission and before peer-review. There can be important differences between the submitted version and the official published version of record.

People interested in the research are advised to contact the author for the final version of the publication, or visit the DOI to the publisher's website.

- The final author version and the galley proof are versions of the publication after peer review.

- The final published version features the final layout of the paper including the volume, issue and page numbers.

Link to publication

\footnotetext{
General rights rights.

- You may freely distribute the URL identifying the publication in the public portal. please follow below link for the End User Agreement:

www.umlib.nl/taverne-license

Take down policy

If you believe that this document breaches copyright please contact us at:

repository@maastrichtuniversity.nl

providing details and we will investigate your claim.
}

Copyright and moral rights for the publications made accessible in the public portal are retained by the authors and/or other copyright owners and it is a condition of accessing publications that users recognise and abide by the legal requirements associated with these

- Users may download and print one copy of any publication from the public portal for the purpose of private study or research.

- You may not further distribute the material or use it for any profit-making activity or commercial gain

If the publication is distributed under the terms of Article $25 \mathrm{fa}$ of the Dutch Copyright Act, indicated by the "Taverne" license above, 


\title{
Efficient Functionalization of Additives at Supramolecular Material Surfaces
}

\author{
Olga J. G. M. Goor, Henk M. Keizer, Anne L. Bruinen, Moniek G. J. Schmitz, \\ Ron M. Versteegen, Henk M. Janssen, Ron M. A. Heeren, and Patricia Y. W. Dankers*
}

Supramolecular polymeric materials are formed by molecular building blocks that are connected through directed noncovalent interactions such as hydrogen bonding, electrostatic, metal-ligand, or $\pi-\pi$ interactions. ${ }^{[1,2]}$ The supramolecular interactions give the materials their dynamic nature, in a similar way as living systems, for example, control the complex process of extracellular matrix assembly, remodeling, and bioactivation. ${ }^{[3]}$ This dynamic nature results in synthetic supramolecular materials with extraordinary mechanical, processable, responsive, modular, and tunable properties that cannot be achieved with conventional macromolecules. ${ }^{[4]}$ Seminal contributions of supramolecular polymers have been reported, showing applicability as strong thermoplastic elastomers, ${ }^{[5,6]}$ functional biomaterials, ${ }^{[7,8]}$ drug-delivery vehicles, ${ }^{[9]}$ self-healing materials, ${ }^{[10-12]}$ light-emitting diodes, and molecular electronics. ${ }^{[13-15]}$

Via a modular approach through co-assembly of several supramolecular constituents, complexity, and functionality can be introduced into supramolecular aggregates and polymeric structures. ${ }^{[4]}$ This modular approach allows for the mixingand-matching of functional additives with various chemical, physical, and/or biological properties. Intrinsically these modified additives influence the assembly, and therefore properties, of the base supramolecular constituents. Only a few examples are disclosed in which functional additives are incorporated in solid-like supramolecular materials, such as the incorporation of supramolecular reinforcement fillers in supramolecular thermoplastic elastomers (TPE). ${ }^{[16]}$

\footnotetext{
O. J. G. M. Goor, M. G. J. Schmitz, Dr. P. Y. W. Dankers Institute for Complex Molecular Systems Eindhoven University of Technology

P.O. Box 513,5600 MB, Eindhoven, The Netherlands E-mail: p.y.w.dankers@tue.nl

O. J. G. M. Goor, M. G. J. Schmitz, Dr. P. Y. W. Dankers Laboratory of Chemical Biology

Department of Biomedical Engineering

P.O. Box 513, 5600 MB, Eindhoven, The Netherlands

Dr. H. M. Keizer, Dr. R. M. Versteegen, Dr. H. M. Janssen

SyMO-Chem B.V.

Het Kraneveld 4, 5612 AZ, Eindhoven, The Netherlands

A. L. Bruinen, Prof. R. M. A. Heeren

M4I, the Maastricht MultiModal Molecular Imaging Institute Maastricht University

Universiteitssingel 50, 6229 ER, Maastricht, The Netherlands

Dr. P. Y. W. Dankers

Laboratory for Cell and Tissue Engineering

Department of Biomedical Engineering

P.O. Box 513, $5600 \mathrm{MB}$, Eindhoven, The Netherlands

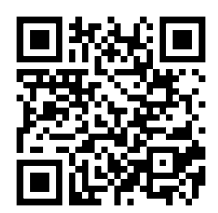

DOI: 10.1002/adma.201604652
In our group we showed that supramolecular TPE can be made using a four-fold hydrogen bonding 2-ureido-4-pyrimidinone (UPy) motif. . $^{[5,17-19]}$ These supramolecular TPE materials could be functionalized via a modular approach by mixing with UPy-functionalized additives. ${ }^{[20,21]}$ Recent developments show applicability of this approach as antifouling agents ${ }^{[22]}$ and bioactive peptides. ${ }^{[23,24]}$ The additives have been simply mixed with the supramolecular TPE to provide the material with functionality, ${ }^{[21]}$ resulting in antifouling materials and cell-adhesive scaffolds. Strikingly, in almost all of these materials, the exact distribution of the supramolecular additive as well as specific surface enhancement cannot be fully controlled, while in several applications solely surface function is required. Moreover, in biomaterials science, the surface functionality is frequently provided by complex bioactive modules, that are often highly incompatible with the material preparation and processing conditions. Decoupling of processing and functionalization strategies offers flexibility in the choice of processing method, and allows for exclusive surface modification.

Many examples have been reported using several chemoselective reaction strategies. ${ }^{[25-36]}$ Covalent postmodification via such a chemoselective reaction is an elegant approach to introduce surface functionality on solid materials. Although functionality can be conveniently introduced on these materials, they are not inherently dynamic in comparison to supramolecular materials. In order to benefit both from the dynamic nature of supramolecular polymers, and the advantageous and wide applicability of postmodification strategies on material surfaces, we report here for the first time covalent modification of supramolecular additives in supramolecular polymeric materials.

To this end, we used the inverse electron demand DielsAlder (iEDDA) cycloaddition ${ }^{[37-42]}$ reaction which has been reported to be the fastest bio-orthogonal reaction in aqueous environment ${ }^{[43,44]}$ as postmodification strategy. Therefore, we designed a supramolecular TPE material in which a reactive UPy-modified tetrazine (UPy-Tz) additive is incorporated, providing a handle for selective surface postmodification via iEDDA (Scheme 1a). Using trans-cyclooctene (TCO) moieties, functionalities are introduced at this supramolecular TPE material surface (Scheme 1c). In conjunction with advanced analysis techniques, the UPy-modified additive distribution and concomitant surface modification is shown. Although materials have been analyzed providing detailed chemical analysis in $2 \mathrm{D}^{[45]}$ and $3 \mathrm{D},{ }^{[46-48]}$ we were able for the first time to resolve in detail the surface and bulk composition of a supramolecular material using time-of-flight secondary-ion mass spectrometry with depth profiling (3D ToF-SIMS). Moreover, we have shown 


\section{Surface Analysis}

Post-Modification

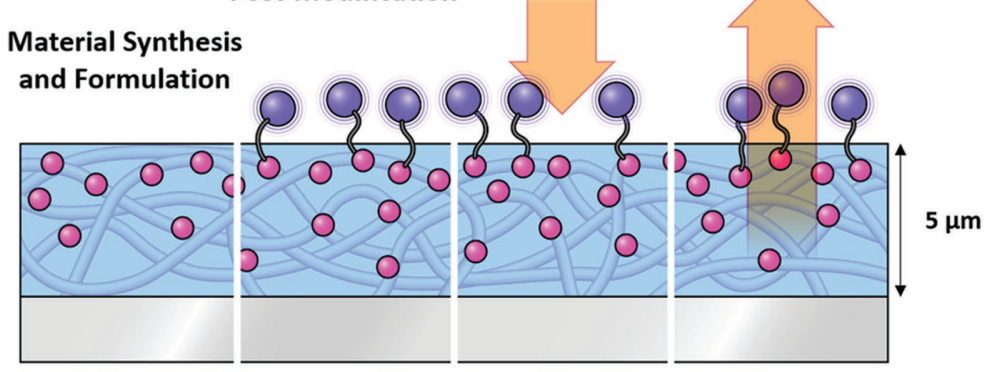

b

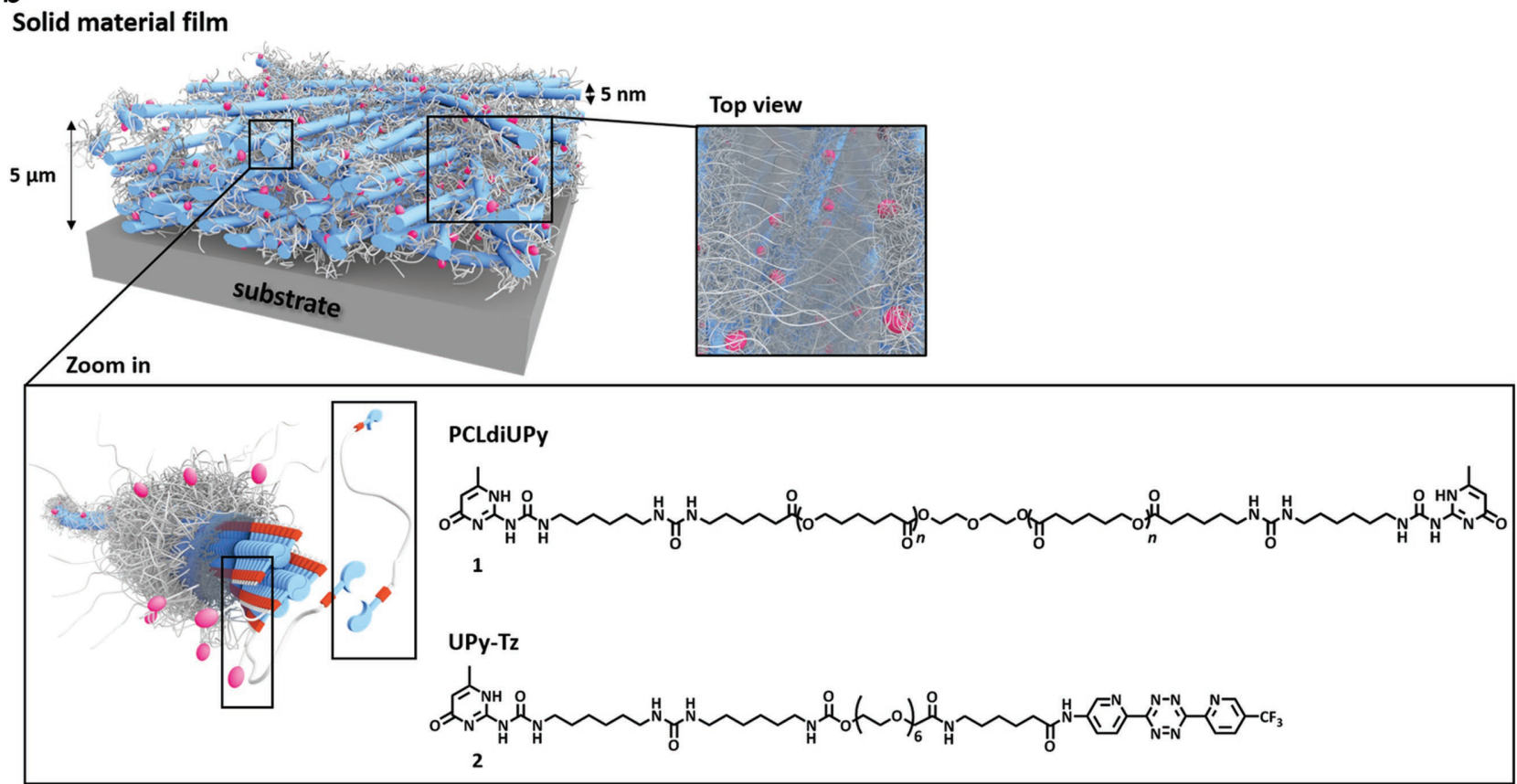

C

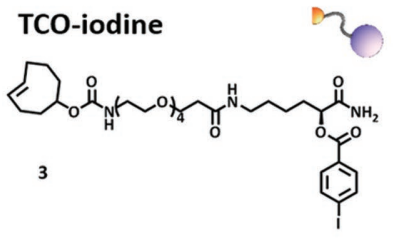

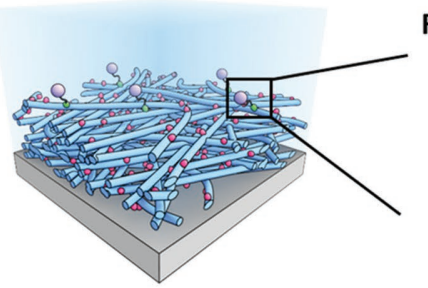

Reaction product

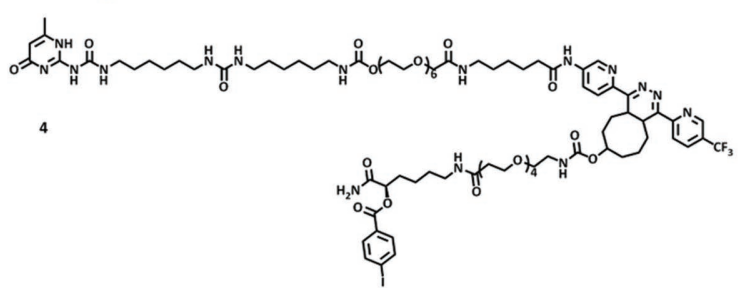

Scheme 1. Schematic representations of the surface modified supramolecular materials. a) Representation of the subsequent steps we take: i) Supramolecular material synthesis and formulation, ii) surface postmodification, iii) surface analysis, and iv) surface and bulk depth profiling by 3D ToFSIMS combined with sequential reconstruction. The drop-cast supramolecular TPE material films are typically $5 \mu \mathrm{m}$ in thickness. b) Representation of the self-assembled structure of the solid supramolecular film. The fibers within this solid network (depicted in blue) are composed of bundles of stacked UPy-UPy moieties in the lateral direction, forming a hard phase of $\approx 5 \mathrm{~nm}$ in diameter. The soft phase is composed of the PCL polymer chains (depicted in gray) filling up the total space in between the hard phase (as is common for traditional thermoplastic elastomers). In a modular fashion, UPy-functionalized tetrazine guest moieties (UPy-Tz, (2), pink dots) can be incorporated in the core of these nanofibers formed by the PCLdiUPy (1) molecules. The top view provides an overview of the solid film from above, demonstrating the dense characteristic of the films. c) Chemical structure of a TCO-iodine (3) with an I atom for detection with XPS (TCO-iodine) that can react at the surface of the supramolecular film resulting in the corresponding reaction product (4). 
decoupling of the processing conditions and post-modification strategy of the materials by first electrospinning the materials and subsequently introducing functionalization via covalent postmodification.

In order to achieve selective surface reaction, analysis and detailed depth profiling, we carefully designed and synthesized a supramolecular UPy-Tz additive (Scheme 1b; Figure S1, Supporting Information), that provides supramolecular intercalation into the $\mathrm{TPE}^{[49]}$ and which proved to be stable up to at least 7 months. The UPy-Tz additive consists of a hydrophilic oligo(ethylene glycol) (OEG) spacer which is proposed to facilitate surface exposure via extension into water. Furthermore, the UPy-Tz molecule is equipped with a $\mathrm{CF}_{3}$ group that allows for detection. As base supramolecular TPE polycaprolactone (PCL) $\left(M_{\mathrm{n}}=2 \mathrm{kDa}\right)$ telechelically modified with UPy-units was used (PCLdiUPy, Scheme 1b). ${ }^{[50]}$ An iodinated TCO-moiety (TCO-iodine) was synthesized to perform the cycloaddition at the surface-liquid interface of the material (Scheme 1c; Figure S3, Supporting Information). Moreover, the generality of this approach was shown by reaction of a TCO-conjugated fluorescent protein reacted at the supramolecular TPE surface as well as the translation to different length scales and processing strategies using electrospinning.

The influence of UPy-Tz addition to PCLdiUPy drop-cast films (preparation details are provided in the Methods section in the Supporting Information) with respect to surface morphology was investigated with AFM (Figure S8, Supporting Information). Fibrous structures were observed up to $5 \mathrm{~mol} \%$ of UPy-Tz, whereas introduction of $10 \mathrm{~mol} \% \mathrm{UPy}-\mathrm{Tz}$ showed the formation of hard domains among the fibrous structures. An increase of the UPy-Tz content to $25 \mathrm{~mol} \%$ results in a fully covered surface with crystalline domains (Figure S8d, Supporting Information). The presence of fibers in the $5 \mathrm{~mol} \%$ UPy-Tz phase image indicates the UPy-Tz is completely mixed with the PCLdiUPy (Figure S8b, Supporting Information), while the hard domains are proposed to be phase separated, crystalline domains of the UPy-Tz (Figure S8d, Supporting Information). In contrast, AFM phase images of spin coated material films show fibrous structures up to $10 \mathrm{~mol} \%$ UPy-Tz incorporation, and phase separation at 25 mol\% UPy-Tz incorporation (Figure S9, Supporting Information). Moreover, the fibers are both thinner and shorter in length in the spin coated samples, which is possibly explained by faster solvent drying during spin coating which reduces assembly time. On a macroscopic level, water contact angle measurements on air dried drop-cast films show an increase in contact angle from $68 \pm 1.1^{\circ}$ for PCLdiUPy to $84 \pm 1.2^{\circ}$ for PCLdiUPy with 25 mol\% UPy-Tz (Table S1, Supporting Information), indicative for an increase in surface hydrophobicity. Surfaces of $100 \%$ UPy-Tz did not result in a smooth film, hence these contact angles could not be determined. XPS measurements revealed fluorine intensities of 0.49, 4.07, and 7.43 at\% for PCLdiUPy with 5, 10, and 25 mol\% UPy-Tz, respectively. Concomitantly an increase in nitrogen intensities was detected: 4.75 at\% for PCLdiUPy, and 5.14, 8.92, and 12.9 at\% for 5, 10, and 25 mol\% UPy-Tz, respectively (Figure 1a-c; Figure S10b, Supporting Information). These results demonstrate that surfaces of the supramolecular films are gradually enriched in both fluorine and nitrogen upon addition of higher amount of UPy-Tz. In addition, also angleresolved XPS (ARXPS) measurements, with angle increments of $15^{\circ}$ to a maximum angle of $75^{\circ}$, pleasingly confirmed an increase in the fluorine to carbon $(\mathrm{F}: \mathrm{C})$ ratio from 0.13 to 0.22 upon increasing resolving angle. Upon increasing resolving angle the relative surface area that is measured is increased, while the penetration depth of the X-ray beam decreases, thus an increase in $\mathrm{F}: \mathrm{C}$ ratio indicates fluorine enrichment at the surface of the supramolecular material film (Figure S10c,d, Supporting Information).

Since the iEDDA cycloaddition postmodification reactions are performed in aqueous solution, it was of interest to investigate the surface composition after water annealing. XPS measurements after $48 \mathrm{~h}$ of water annealing showed an increase in fluorine intensity from 4.36 at\% (PCLdiUPy with $5 \mathrm{~mol} \%$ UPy-Tz) up to 9.65 at\% (PCLdiUPy with 25 mol\% UPy-Tz) as well as nitrogen from 4.82 at\% for PCLdiUPy to 16.2 at\% for PCLdiUPy with 25 mol\% UPy-Tz (Figure S10b, Supporting Information) compared to air dried samples. We hypothesize that this enhanced stratification might favor the reaction of the TCO-moiety at the surface-water interface. Subsequent investigation of the drop-cast films using FTIR revealed a gradual increase in stretch vibration around $1330 \mathrm{~cm}^{-1}$ upon increasing amounts of UPy-Tz incorporation, which is attributed to the typical C-F bond vibration found in the $1400-1100 \mathrm{~cm}^{-1}$ region, ${ }^{[51]}$ confirming the presence of UPy-Tz at the surface of the material film (Figure 1d). In conclusion, surface analysis both on a macroscopic level as well as on the molecular level revealed UPy-Tz presence at the material surface and moreover demonstrated further enrichment after water annealing. Additionally, we showed that the UPy-Tz additive is mixed into the supramolecular TPE, and that the material properties are tunable, i.e., accessibility of UPy-Tz for reaction, based on the amount that was mixed in. Therefore, we propose these moieties to be perfectly suitable for selective surface functionalization of supramolecular materials.

The presence of the UPy-Tz at the surface of the films suggests convenient surface modification using a TCOmodified molecule as reagent (Scheme 1c). Upon reaction of the TCO-iodine with the UPy-Tz the pink color of the tetrazine significantly decreases. This corresponds to a decrease in the characteristic tetrazine absorption band at $540 \mathrm{~nm}$, after the 1,2-diazine click product has been formed and $\mathrm{N}_{2}$ is released, ${ }^{[52]}$ indicative for the reaction to have occurred and the enhanced presence of the UPy-Tz at the material surface (Figure S15, Supporting Information). Furthermore, after reaction with TCO-iodine, water contact angle measurements revealed increased surface hydrophobicity upon incorporation of higher amounts of UPy-Tz with angles of $63.4 \pm 5.0^{\circ}$ for PCLdiUPy up to $75.8 \pm 1.1^{\circ}$ for PCLdiUPy containing $25 \mathrm{~mol} \% \mathrm{UPy}-\mathrm{Tz}$ (Table S1, Supporting Information).

Importantly, XPS measurements are expected to show an increase of an additional iodine peak in line with the increase in fluorine peak intensity upon the addition of higher amounts of UPy-Tz (Figure 1e-g). The spectrum of the reference surface, i.e., pristine PCLdiUPy, shows an iodine signal of 0.12 at\% suggesting nonspecific adsorption of the TCO-iodine, which is attributed to the hydrophobic nature of the surface (Figure 1f). Upon the incorporation of 5 and $10 \mathrm{~mol} \% \mathrm{UPy}-\mathrm{Tz}$, an increase 

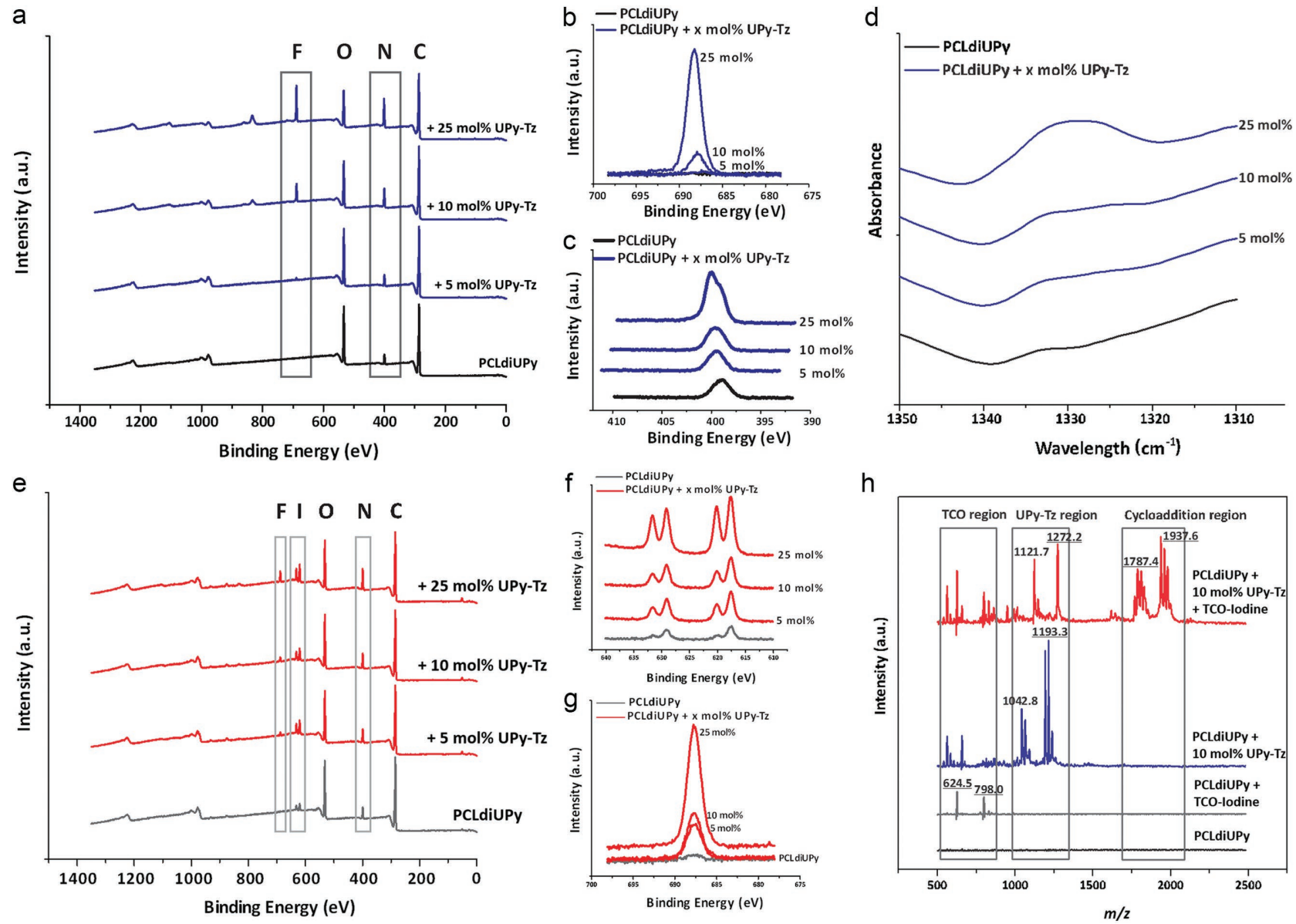

Figure 1. Surface analyses of the supramolecular drop-cast films with UPy-Tz additive and subsequent postmodification with TCO-iodine. a) XPS results of the different films. Increase in $\mathrm{F}$ and $\mathrm{N}$ peaks are highlighted. b) XPS zoom of the characteristic $\mathrm{F}$ binding energy area for the different films. c) XPS zoom of the characteristic $\mathrm{N}$ binding energy area for the different films. d) FTIR zoom in of the characteristic C-F vibration stretch for the different films. e) XPS overview of the different films after postmodification with TCO-iodine. Increase in F, I, and N peaks is highlighted. $\mathrm{f}$ ) XPS zoom of the characteristic I binding energy area for the different films. g) XPS zoom of the characteristic F binding energy area for the different films. h) Surface MALDI-ToF MS analysis of PCLdiUPy (black line), PCLdiUPy incubated with TCO-iodine (gray line), PCLdiUPy with 10 mol\% UPy-Tz (blue line), and PCLdiUPy with 10 mol\% UPy-Tz incubated with TCO-iodine (red line).

in iodine intensity to 0.34 at $\%$ is observed in both cases, indicative for a successful reaction at the surface. The incorporation of 25 mol\% UPy-Tz shows a further increase to 0.72 at\% iodine, indicating more TCO-iodine is reacted at this materials surface.

Surface MALDI-ToF MS studies were performed to directly confirm the presence of the reaction product (Figure 1h). The mass spectrum of the control PCLdiUPy surface without TCO-iodine (Figure 1h; black spectrum) does not show any signal, which is proposed to result from the matrix solvent (acetonitrile) to suppress polymer signals. PCLdiUPy incubated with TCO-iodine (Figure 1h; gray spectrum) shows the TCO-iodine mass $(\mathrm{m} / z$ 798.02) and a cleaved TCO-iodine adduct which corresponds to a fragment from which $\mathrm{CO}_{2}$ is released $(\mathrm{m} / z$ 624.45). The surface of PCLdiUPy with $10 \mathrm{~mol} \%$ UPy-Tz incorporated that was not incubated with TCO-iodine (Figure 1h; blue spectrum) presents a few matrix peaks in the TCO region. Additionally, in the UPy-Tz region the UPy-Tz mass $(m / z 1190.29)$ and the corresponding salt adducts are visible. Besides that, a UPy-Tz fragment resulting from cleavage of the urea bond next to the UPy-moiety $(\mathrm{m} / z$ 1042.59) is present as well. The surface of PCLdiUPy with $10 \mathrm{~mol} \% \mathrm{UPy}-\mathrm{Tz}$ incorporated that was incubated with TCO-iodine (Figure 1h; red spectrum) clearly presents the cycloaddition click product $(m / z$ 1937.68) with the corresponding salt adducts. Moreover, the cycloaddition product where the UPy-moiety is cleaved $(m / z 1787.52)$ was observed. Furthermore, two additional peaks are present that correspond to the mass of the cycloaddition product after release of $\mathrm{CO}_{2}$ from the TCO-iodine (left peaks, cleaved UPy, $m / z$ 1122.05; right peaks, intact UPy, $m / z$ 1272.30), indicating that all UPy-Tz has reacted. Surface MALDI-ToF MS of PCLdiUPy with 5 and 25 mol\% UPy-Tz show similar trends (Figure S11, Supporting Information). Moreover, the shift of the peaks in the UPy-Tz region (Figure $1 \mathrm{~h}$ ) demonstrates the efficiency of the surface modification, as no unreacted UPy-Tz was observed after the reaction. Accordingly, surface MALDIToF MS measurements revealed indisputable evidence for the successful click reaction at the surface of the supramolecular material surface. 
To the best of our knowledge, we for the first time report on the spatial characterization of the surface and bulk composition of supramolecular spin-coated polymer films, using a series of 50 subsequent surface analyses and sputter events in negative ion mode. Chemical structures of the mass fragments discussed here are depicted in Figure S12 in the Supporting Information. 3D reconstruction convincingly shows a gradual increase in fluorine content throughout the material film upon increasing amounts of UPy-Tz (Figure 2a-d; mass spectra of the surface are depicted in Figure S13 in the Supporting Information). For relatively low amounts of UPy-Tz (i.e., 1 and $5 \mathrm{~mol} \%$ ) the fluorine predominantly appears at the surface of the material film, whereas at high amounts (i.e., 10 and $25 \mathrm{~mol} \%$ ) the fluorine is also distributed throughout the bulk of the material. 3D reconstruction concomitantly reveals an increase in iodine content at the material surface (Figure 2a-d) demonstrating occurrence of the reaction at the surface. Distribution profiles of UPy-fragments $(\mathrm{m} / z 124$ and 150, respectively) and monomers of the PCL $(m / z 113)$ showed a homogeneous distribution throughout the films (Figure 2e-h). Upon detailed total-ion-count analyses of the different conditions $(1,5,10$, and $25 \mathrm{~mol} \% \mathrm{UPy}-\mathrm{Tz}$, respectively) both at the surface and in the bulk (Figure 2i) we a

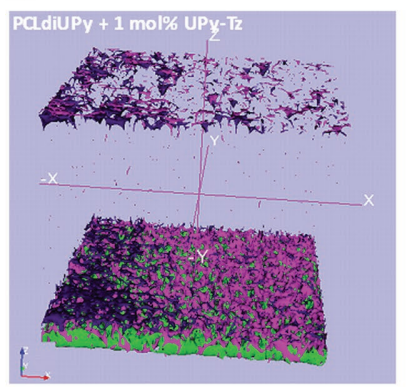

e

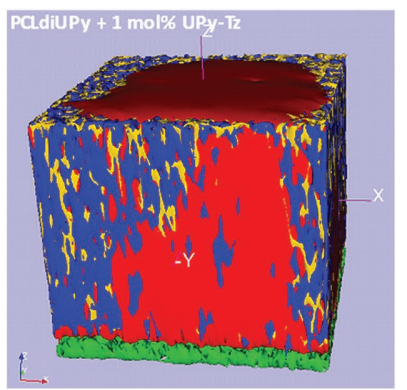

$\mathrm{b}$

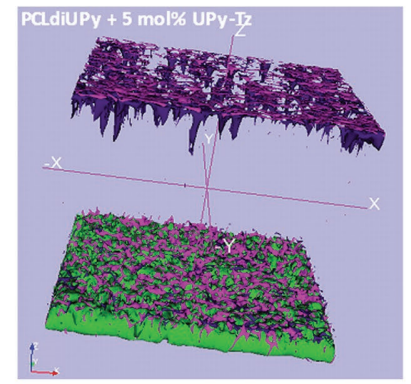

f

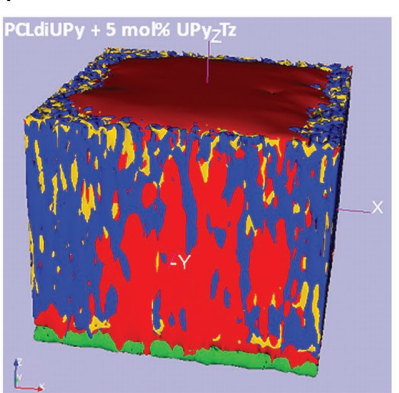

C

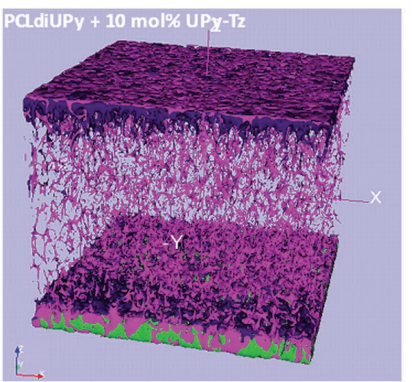

g

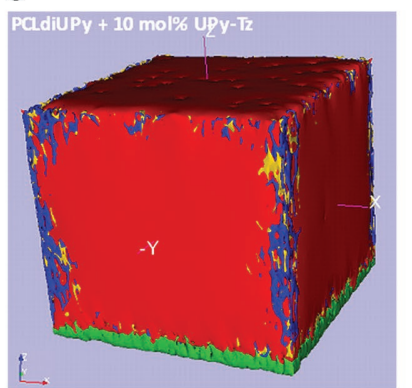

d

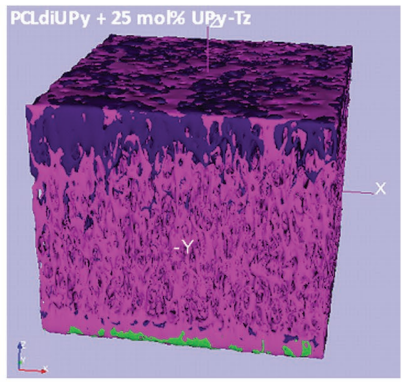

$\mathrm{h}$

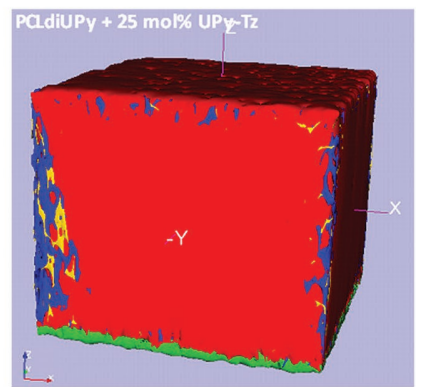

i

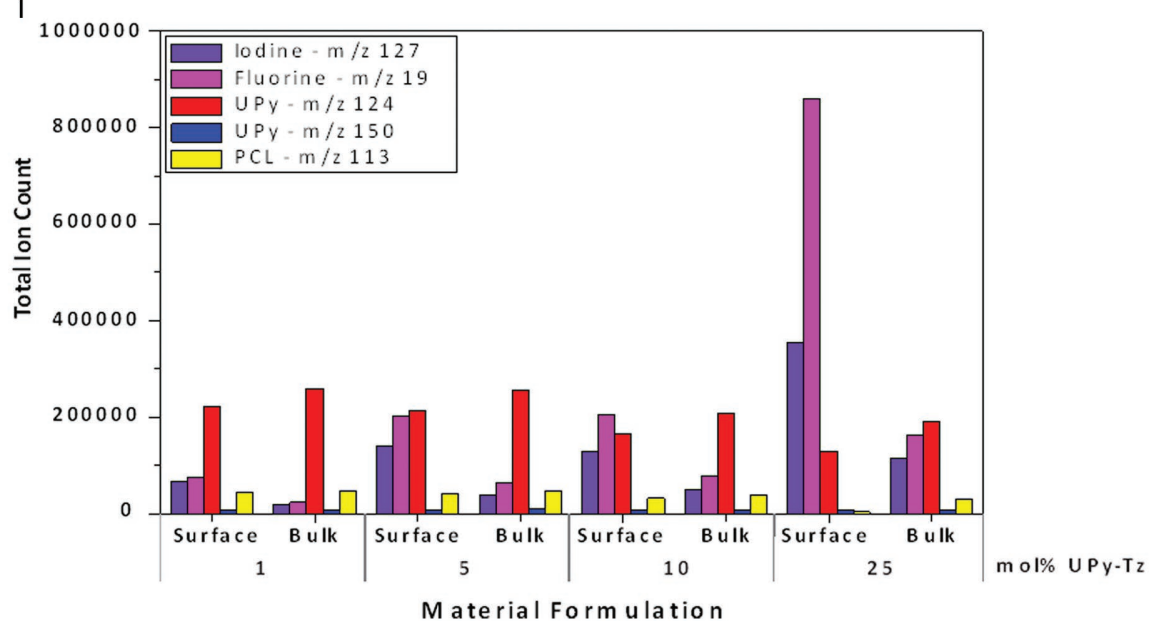

Figure 2. 3D depth profile of supramolecular spin-coated films after postmodification using ToF-SIMS. The films have a thickness of 100-150 nm. Relevant mass fragments are depicted in different colors: iodine $=$ purple, fluorine $=$ pink, UPy-fragment $m / z 124=$ red, UPy-fragment $m / z 150=$ blue, PCL-fragment $=$ yellow, ITO $=$ green. Dimensions of depth profile area are $100 \mu \mathrm{m} \times 100 \mu \mathrm{m}$. Reconstructions were made after 50 sputter cycles of $1 \mathrm{~min}$ with $20 \mathrm{keV} \mathrm{C} 60^{+}$sputter beam and subsequent surface analysis of four frames $(2.13 \mathrm{~min})$ using a $30 \mathrm{keV} \mathrm{Bi}{ }_{3}{ }^{++}$beam. Spatial distribution of $\mathrm{I}$ and $\mathrm{F}$ ions, and ITO fragment in films of PCLdiUPy with: a) 1 mol\% UPy-Tz, b) 5 mol\% UPy-Tz, c) 10 mol\% UPy-Tz, and d) 25 mol\% UPy-Tz. Spatial distribution of two UPy-fragments, PCL-fragment, and ITO fragment in films of PCLdiUPy with: e) 1 mol\% UPy-Tz, f) 5 mol\% UPy-Tz, g) 10 mol\% UPyTz, and h) 25 mol\% UPy-Tz. i) Total ion count plot of all masses of interest, analyzed for both the surface and the bulk. 
observed that in all cases total ion counts of both fluorine and iodine decreased in bulk as compared to the surface. Additionally, a general trend in the increase of the fluorine and the iodine content at the surface upon higher UPy-Tz amounts was measured, whereas both UPy and PCL ion counts remain constant (Figure 2i). Interestingly, principal component analysis (PCA) of the surface of PCLdiUPy with 1 and 5 mol\% UPy-Tz, respectively, reacted with TCO-iodine showed a correlation between fluorine and iodine ( +1 principal component), and between the UPy-fragments and the PCL-fragments $(-1$ principal component) (Figure S14a-f, Supporting Information).

Pleasingly 3D ToF-SIMS imaging with $\mathrm{C}_{60}{ }^{+}$depth profiling has provided useful and detailed insight into the chemical composition of our materials, both at the surface and in bulk. Due to the high depth resolution, this technique holds immense promise in a variety of material science developments, where control and understanding of the polymer structures is extremely important for their function. This technique now paves the way for high resolution analysis of materials in $3 \mathrm{D}$ and thereby allows for specific molecular design in order to meet material requirements for a variety of applications.

Post-modification of our supramolecular material surfaces with more complex molecules, such as proteins, demonstrates the generality of our approach. Therefore, we investigated the cycloaddition click reaction of enhanced yellow fluorescent protein (EYFP) (5) to UPy-Tz by fluorescence spectroscopy (Figure S16, Supporting Information). EYFP was a

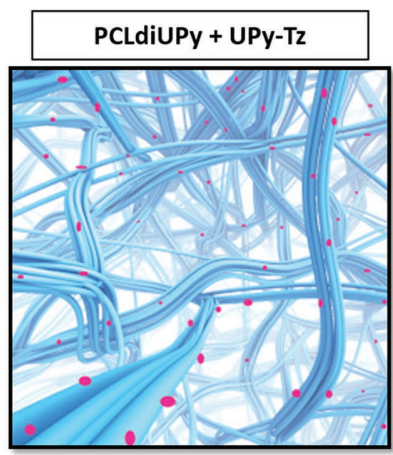

d

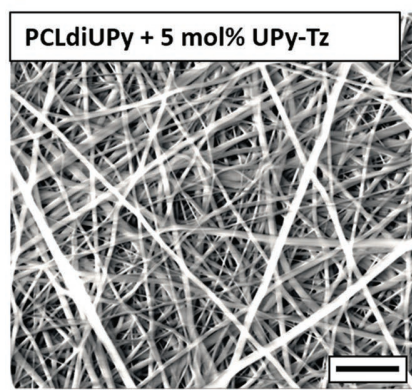

g

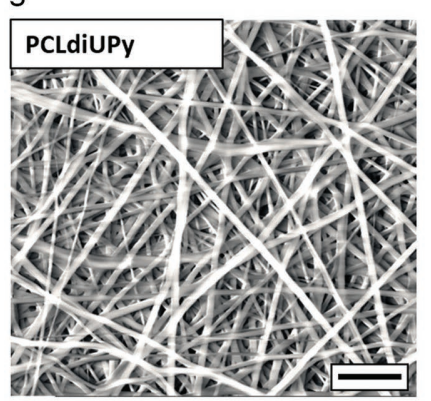

b

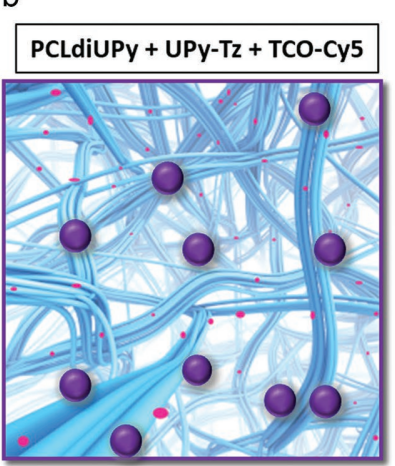

e

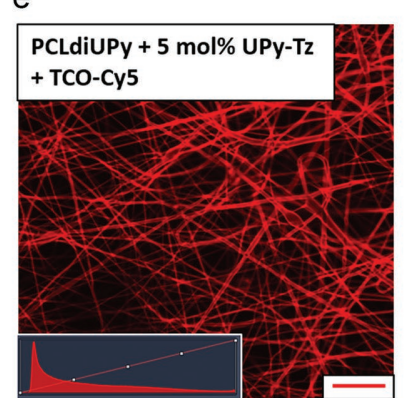

$\mathrm{h}$

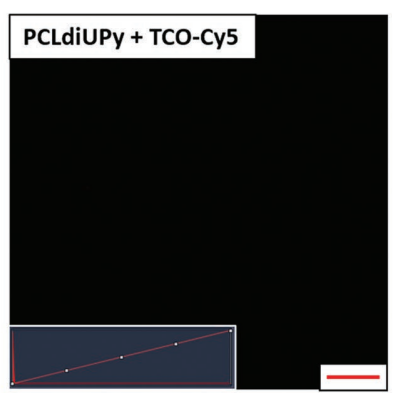

c

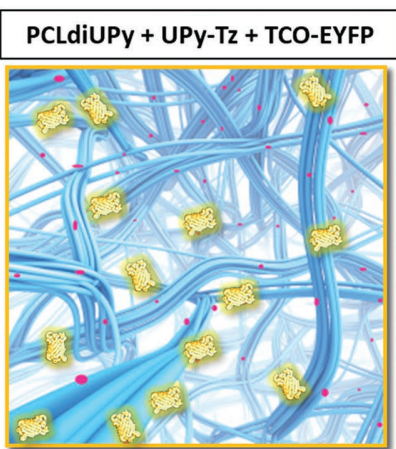

f
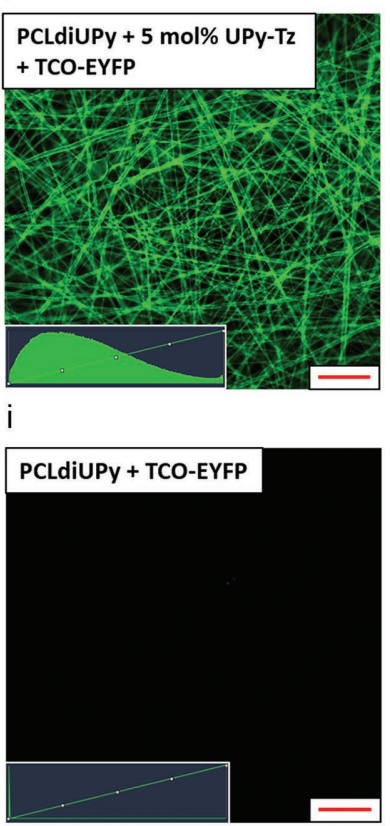

Figure 3. Decoupling processing and functionalization of electrospun supramolecular polymer materials. a) Cartoon representation of the electrospun supramolecular materials consisting of PCLdiUPy as the base polymer and UPy-Tz (pink dots) incorporated to facilitate selective surface reaction, b) reacted with the TCO-Cy5 dye, or c) reacted with the TCO-EYFP model protein. d) SEM picture of electrospun fibers of PCLdiUPy with 5 mol\% UPy-Tz incorporated, diameters $0.6 \pm 0.24 \mu \mathrm{m}$, and the control electrospun fibers of PCLdiUPy $(\mathrm{g})$, diameters $0.8 \pm 0.36 \mu \mathrm{m}$, scale bars represent $10 \mu \mathrm{m}$. e) Confocal image of electrospun fibers of PCLdiUPy with 5 mol\% UPy-Tz incorporated and the PCLdiUPy control (h), incubated with $1 \mathrm{mg} \mathrm{mL}^{-1} \mathrm{TCO}$ Cy5, fibers show a clear red color, scale bar represents $20 \mu \mathrm{m}$, inset shows the fluorescence intensity plot. f) Confocal image of electrospun fibers of PCLdiUPy with 5 mol\% UPy-Tz incorporated and the PCLdiUPy control (i), incubated with $1 \mathrm{mg} \mathrm{mL} \mathrm{mL}^{-1} \mathrm{TCO}$-EYFP, fibers show a clear green color, scale bar represents $20 \mu \mathrm{m}$, inset shows the fluorescence intensity plot. 
nonspecifically functionalized with TCO-moieties (TCO-EYFP) (Figure S7, Supporting Information). Incorporation of 1 and $5 \mathrm{~mol} \%$ UPy-Tz showed a 9 times increase in surface fluorescence after TCO-EYFP incubation, whereas for both 10 and 25 mol\% UPy-Tz 4 times and 2 times increase, respectively, was observed. This moderate increase for larger mol\% of UPy-Tz can be explained by differences in morphology of the different mixtures, i.e., the occurrence of phase separated domains at the surface (Figure S8, Supporting Information). It is proposed that in these cases tetrazine moieties are less available for reaction. An additional explanation might be that a higher amount of protein is reacted on the PCLdiUPy with 10 and $25 \mathrm{~mol} \%$ UPy-Tz surfaces, thereby inducing quenching of the fluorophores. Surfaces incubated with nonfunctionalized EYFP did not show any fluorescence indicating the EYFP is washed off. In conclusion, these protein conjugation experiments show the versatility of our strategy toward more complex surface modifications.

Electrospun meshes of PCLdiUPy with $5 \mathrm{~mol} \% \mathrm{UPy}-\mathrm{Tz}$ were prepared (Figure 3a), and PCLdiUPy as control. After electrospinning the materials were incubated with either a TCO-conjugated Cy5 dye (4) (Figure 3b; Figure S5, Supporting Information) or a TCO-EYFP protein (Figure $3 \mathrm{c}$ ). Scanning electron microscopy images show fiber formation for both the PCLdiUPy with 5 mol\% UPy-Tz (Figure 3d) and the PCLdiUPy (Figure $3 \mathrm{~g}$ ) with fiber diameters of $0.6 \pm 0.24$ and $0.8 \pm 0.36 \mu \mathrm{m}$, respectively. Functionalization with the TCO-Cy 5 dye showed clear red appearance of the fibers (Figure 3e) for the PCLdiUPy with $5 \mathrm{~mol} \%$ UPy-Tz materials whereas the PCLdiUPy (Figure 3h) materials did not show any fluorescence. A similar trend was observed after incubation with the TCOEYFP, the PCLdiUPy with 5 mol\% UPy-Tz (Figure 3f) showed green fiber morphology while the PCLdiUPy materials did not yield any fluorescence (Figure 3i). Moreover, higher magnification images (Figure S17, Supporting Information) clearly showed enhanced fluorescence at the surface of the electrospun fibers, indicative for the reaction to have occurred at the surface. This approach shows decoupling of the material processing conditions and subsequent material functionalization via a postmodification approach.

Here we show that selective modification reactions can be performed on additives that are supramolecularly incorporated into supramolecular materials. Careful design and synthesis of the additive facilitates control on the assembly process within the material and allows for selective surface postmodification in aqueous environment. Importantly, in this way processing of the material, that regularly requires harsh processing conditions (i.e., the use of organic solvents and/or high temperatures), and functionalization can be decoupled. Along these lines this approach endorses various material preparation methods (i.e., 3D-printing, melt spinning, electrospinning), whereby bulk material properties can easily be tuned and yet the material surfaces can be functionalized via covalent postmodification. Here we have shown the feasibility of this approach by decoupling of material processing by electrospinning and postmodification of the supramolecular additive via selective orthogonal surface reactions. In conjunction with advanced ToF-SIMS depth profiling of bulk and surface we are now able to reveal the distribution of additive and reactant.
At a supramolecular chemistry level these results allow for further exploration of these selective reactions on inherently more dynamic systems, such as aggregates in solution and supramolecular hydrogels, yielding complex assemblies with various functionalities. This postmodification strategy of supramolecular systems might accommodate for better control on the assembled structure, when specific functionality is introduced after assembly. Additionally, the accurate depth resolution of ToF-SIMS is proposed to aid in elucidating the complex structures of such hierarchical assemblies.

Finally, this bio-orthogonal functionalization and characterization strategy holds great promise in the field of regenerative medicine, in which design and detailed analysis of bioactive, functional biomaterials is important for the ultimate interaction with cells and tissues, and the essential performance. As a result of the bio-orthogonal nature, the supramolecular thermoplastic elastomeric materials might as well be suitable for in vivo functionalization.

\section{Supporting Information}

Supporting Information is available from the Wiley Online Library or from the author.

\section{Acknowledgements}

The authors thank E. W. Meijer for critical reading of the manuscript and useful discussions on the results, J. L. J. van Dongen and X. Lou for helpful discussion regarding analyses, M. W. G. M. Verhoeven and R. J. Lancee for XPS collaboration, G. B. Eijkel for assistance regarding ToF-SIMS depth profiling experiments, D. J. Mulder for assistance with FTIR, R. P. G. Bosmans for collaboration during protein expression and the ICMS Animation Studio for careful design of cartoons. This work was financially supported by the European Research Council (FP7/20072013) ERC Grant Agreement 308045 and the Ministry of Education, Culture and Science (Gravity Program 024.001.03).

Received: August 30, 2016 Revised: October 11, 2016 Published online: November 29, 2016

[1] J.-M. Lehn, Polym. Int. 2002, 51, 825

[2] G. M. Whitesides, B. Grzybowski, Science 2002, 295, 2418.

[3] R. J. Epstein, Human Molecular Biology: An Introduction to the Molecular Basis of Health and Disease, Cambridge University Press, Cambridge, UK 2003.

[4] T. Aida, E. W. Meijer, S. I. Stupp, Science 2012, 335, 813.

[5] B. J. B. Folmer, R. P. Sijbesma, R. M. Versteegen, J. a. J. van der Rijt, E. W. Meijer, Adv. Mater. 2000, 12, 874.

[6] A. W. Bosman, R. P. Sijbesma, E. W. Meijer, Mater. Today 2004, 7, 34.

[7] K. Rajangam, H. A. Behanna, M. J. Hui, X. Han, J. F. Hulvat, J. W. Lomasney, S. I. Stupp, Nano Lett. 2006, 6, 2086.

[8] V. M. Tysseling-Mattiace, V. Sahni, K. L. Niece, D. Birch, C. Czeisler, M. G. Fehlings, S. I. Stupp, J. A. Kessler, J. Neurosci. 2008, 28, 3814.

[9] Y. Bae, S. Fukushima, A. Harada, K. Kataoka, Angew. Chem., Int. Ed. 2003, 42, 4640.

[10] P. Cordier, F. Tournilhac, C. Soulié-Ziakovic, L. Leibler, Nature 2008, 451,977 
[11] M. Burnworth, L. Tang, J. R. Kumpfer, A. J. Duncan, F. L. Beyer, G. L. Fiore, S. J. Rowan, C. Weder, Nature 2011, 472, 334.

[12] Q. Wei, C. Schlaich, S. Prévost, A. Schulz, C. Böttcher M. Gradzielski, Z. Qi, R. Haag, C. A. Schalley, Adv. Mater. 2014, 26, 7358.

[13] J. P. Hill, W. Jin, A. Kosaka, T. Fukushima, H. Ichihara T. Shimomura, K. Ito, T. Hashizume, N. Ishii, T. Aida, Science 2004, 304, 1481.

[14] L. N. Lucas, J. J. D. de Jong, J. H. van Esch, R. M. Kellogg, B. L. Feringa, Eur. J. Org. Chem. 2003, 2003, 155.

[15] R. Abbel, C. Grenier, M. J. Pouderoijen, J. W. Stouwdam, P. E. L. G. Leclère, R. P. Sijbesma, E. W. Meijer, A. P. H. J. Schenning, J. Am. Chem. Soc. 2009, 131, 833.

[16] E. Wisse, L. E. Govaert, H. E. H. Meijer, E. W. Meijer, Macromolecules 2006, 39, 7425

[17] R. P. Sijbesma, F. H. Beijer, L. Brunsveld, B. J. Folmer J. H. Hirschberg, R. F. Lange, J. K. Lowe, E. W. Meijer, Science 1997, 278, 1601.

[18] F. H. Beijer, R. P. Sijbesma, H. Kooijman, A. L. Spek, E. W. Meijer, J. Am. Chem. Soc. 1998, 120, 6761.

[19] R. E. Kieltyka, A. C. H. Pape, L. Albertazzi, Y. Nakano, M. M. C. Bastings, I. K. Voets, P. Y. W. Dankers, E. W. Meijer, J. Am. Chem. Soc. 2013, 135, 11159.

[20] P. Y. W. Dankers, M. C. Harmsen, L. A. Brouwer, M. J. A. Van Luyn, E. W. Meijer, Nat. Mater. 2005, 4, 568

[21] E. Wisse, A. J. H. Spiering, P. Y. W. Dankers, B. Mezari, P. C. M. M. Magusin, E. W. Meijer, J. Polym. Sci., Part A: Polym. Chem. 2011, 49, 1764.

[22] G. C. van Almen, H. Talacua, B. D. Ippel, B. B. Mollet, M. Ramaekers, M. Simonet, A. I. P. M. Smits, C. V. C. Bouten, J. Kluin, P. Y. W. Dankers, Macromol. Biosci. 2015, 16, 350.

[23] B. B. Mollet, M. Comellas-Aragonès, A. J. H. Spiering, S. H. M. Söntjens, E. W. Meijer, P. Y. W. Dankers, J. Mater. Chem. B 2014, 2, 2483 .

[24] D. E. P. Muylaert, G. C. van Almen, H. Talacua, J. O. Fledderus, J. Kluin, S. I. S. Hendrikse, J. L. J. van Dongen, E. Sijbesma, A. W. Bosman, T. Mes, S. H. Thakkar, A. I. P. M. Smits, C. V. C. Bouten, P. Y. W. Dankers, M. C. Verhaar, Biomaterials 2016, 76, 187.

[25] Z. P. Tolstyka, W. Richardson, E. Bat, C. J. Stevens, D. P. Parra J. K. Dozier, M. D. Distefano, B. Dunn, H. D. Maynard, ChemBioChem 2013, 14, 2464.

[26] J. Zheng, K. Liu, D. H. Reneker, M. L. Becker, J. Am. Chem. Soc. 2012, 134, 17274.

[27] P. M. Imbesi, C. Fidge, J. E. Raymond, S. I. Cauët, K. L. Wooley, ACS Macro Lett. 2012, 1, 473.

[28] T. N. Gevrek, T. Bilgic, H.-A. Klok, A. Sanyal, Macromolecules 2014, $47,7842$.
[29] C. F. Hansell, P. Espeel, M. M. Stamenović, I. A. Barker, A. P. Dove, F. E. Du Prez, R. K. O'Reilly, J. Am. Chem. Soc. 2011, 133, 13828

[30] R. J. Williams, I. A. Barker, R. K. O'Reilly, A. P. Dove, ACS Macro Lett. 2012, 1, 1285.

[31] S. Liu, H. Zhang, R. A. Remy, F. Deng, M. E. Mackay, J. M. Fox, X. Jia, Adv. Mater. 2015, 27, 2783.

[32] N. K. Devaraj, R. Weissleder, Acc. Chem. Res. 2011, 44, 816.

[33] N. K. Devaraj, R. Weissleder, S. A. Hilderbrand, Bioconjug. Chem. 2008, 19, 2297.

[34] F. Lin, J. Yu, W. Tang, J. Zheng, S. Xie, M. L. Becker, Macromolecules 2013, 46, 9515.

[35] J. Zheng, G. Hua, J. Yu, F. Lin, M. B. Wade, D. H. Reneker M. L. Becker, ACS Macro Lett. 2015, 4, 207.

[36] Z. N. Mahmoud, S. B. Gunnoo, A. R. Thomson, J. M. Fletcher, D. N. Woolfson, Biomaterials 2011, 32, 3712.

[37] R. Huisgen, Angew. Chem., Int. Ed. Engl. 1963, 2, 633.

[38] R. Huisgen, Angew. Chem., Int. Ed. Engl. 1963, 2, 565.

[39] H. C. Kolb, M. G. Finn, K. B. Sharpless, Angew. Chem., Int. Ed. 2001, 40, 2004.

[40] R. Rossin, S. M. J. van Duijnhoven, T. Läppchen, S. M. van den Bosch M. S. Robillard, Mol. Pharm. 2014, 11, 3090.

[41] M. L. Blackman, M. Royzen, J. M. Fox, J. Am. Chem. Soc. 2008, 130, 13518

[42] I. A. Barker, D. J. Hall, C. F. Hansell, F. E. Du Prez, R. K. O'Reilly, A. P. Dove, Macromol. Rapid Commun. 2011, 32, 1362.

[43] F. Thalhammer, U. Wallfahrer, J. Sauer, Tetrahedron Lett. 1990, 31 6851.

[44] M. T. Taylor, M. L. Blackman, O. Dmitrenko, J. M. Fox, J. Am. Chem. Soc. 2011, 133, 9646

[45] F. Yang, S. Cho, G. Sun, S. V. Verkhoturov, J. W. Thackeray, P. Trefonas, K. L. Wooley, E. A. Schweikert, Surf. Interface Anal. 2015, 47, 1051

[46] M. Taylor, D. Scurr, M. Lutolf, L. Buttery, M. Zelzer, M. Alexander, Biointerphases 2016, 11, 02A301.

[47] J. Bailey, R. Havelund, A. G. Shard, I. S. Gilmore, M. R. Alexander J. S. Sharp, D. J. Scurr, ACS Appl. Mater. Interfaces 2015, 7, 2654.

[48] T. K. Claus, B. Richter, V. Hahn, A. Welle, S. Kayser, M. Wegener, M. Bastmeyer, G. Delaittre, C. Barner-Kowollik, Angew. Chem., Int. Ed. 2016, 55, 3817.

[49] H. Kautz, D. J. M. van Beek, R. P. Sijbesma, E. W. Meijer, Macromolecules 2006, 39, 4265.

[50] W. P. J. Appel, G. Portale, E. Wisse, P. Y. W. Dankers, E. W. Meijer, Macromolecules 2011, 44, 6776.

[51] G. Socrates, Infrared and Raman Characteristic Group Frequencies: Tables and Charts, John Wiley \& Sons, Chichester, UK 2004.

[52] W. Chen, D. Wang, C. Dai, D. Hamelberg, B. Wang, Chem. Commun. 2012, 48, 1736. 